\title{
Emergency point-of-care ultrasound in Canadian pediatric emergency fellowship programs: current integration and future directions
}

\author{
Julia Hoeffe, MD*; Marie Pier Desjardins, $\mathrm{MD}^{*}$; Jason Fischer, $\mathrm{MD}^{\dagger}$; Benoit Carriere, $\mathrm{MD}^{*}$; \\ Jocelyn Gravel, MD*
}

\section{ABSTRACT}

Background: Adult and pediatric emergency physicians have been using point-of-care ultrasound (POCUS) for many years. It is a fast, usually painless, noninvasive diagnostic tool that does not expose the patient to radiation. Information about its current implementation in pediatric emergency medicine (PEM) fellowship programs in Canada is lacking.

Objective: The main goal of our study was to investigate current integration and future direction of POCUS training in Canadian PEM programs.

Methods: The study consisted of two surveys designed for fellows and program directors of all Canadian PEM fellowship programs. The major aspects of the survey were 1) to describe current training in POCUS in fellowship programs, 2) to compare the kind of training that programs offer with what fellows actually receive, and 3) a needs assessment by fellows and program directors for future POCUS training programs. Surveys were sent to program directors and passed on to their fellows. Results: Ninety percent of fellowship program directors as well as $70 \%(42 / 60)$ of fellows responded to the survey. A formal POCUS curriculum exists in five of the nine PEM programs included in this study. Three programs offer specific pediatric POCUS training. The main application is the FAST (focused assessment with sonography for trauma) exam.

Conclusion: There is a wide variation in POCUS content and delivery across PEM fellowship programs, as well as differences in perceptions of current training and of needs by fellows and program directors. However, given that both groups feel POCUS is very important and essential for PEM training, the opportunity exists to develop a standardized curriculum across Canada.

\section{RÉSUMÉ}

Contexte: Les médecins en urgence adulte et en urgence pédiatrique utilisent depuis de nombreuses années l'échographie au département d'urgence (EDU). II s'agit d'un examen diagnostic non invasif, rapide et généralement indolore, qui n'expose pas les patients à des rayons $\mathrm{x}$. Toutefois, I'on en connaît peu sur la place qu'occupe actuellement l'examen dans les programmes en médecine d'urgence pédiatrique (MUP) au Canada.

Objectif: L'étude avait pour but principal d'examiner la place qu'occupe actuellement la formation en EDU dans les programmes en MUP et son orientation future.

Méthode: L'étude consistait en deux enquêtes, I'une conçue à l'intention des résidents et l'autre, à l'intention des directeurs de programme en urgence pédiatrique au Canada. L'enquête avait pour objectifs principaux de : 1) décrire la formation actuelle en EDU dans les programmes, 2) comparer la formation offerte avec la formation réellement obtenues par les résidents, et 3 ) procéder à une évaluation des besoins par les résidents et les directeurs de programme en ce qui concerne les programmes futurs de formation en EDU. Les questionnaires ont été envoyés aux directeurs de programme, qui eux-mêmes les ont transmis aux résidents.

Résultats: Le taux de réponse a atteint $90 \%$ parmi les directeurs de programme en urgence pédiatrique et $70 \%$ (42/60) parmi les résidents. Sur les neuf programmes d'études en MUP compris dans l'enquête, cinq offrent un programme structuré de formation en EDU, dont trois comportent une formation particulière en EDU pédiatrique. La principale application consiste en l'examen FAST (focused assessment with sonography in trauma).

Conclusions: II existe des différences importantes entre les programmes en urgence pédiatrique en ce qui concerne le contenu et la présentation des cours en EDU, ainsi qu'en ce qui concerne la perception des résidents et des directeurs de programme à l'égard de la formation actuelle et des besoins. Toutefois, comme les deux groupes s'accordent sur le fait que I'EDU est un examen très important et qu'il constitue un élément essentiel de la formation en MUP, il y a là la

From the *Division of Emergency Medicine, Department of Pediatrics, CHU Sainte-Justine, Université de Montréal, Montréal, QC; and †Division of Emergency Medicine, Department of Pediatrics, The Hospital for Sick Children, Toronto, ON.

Correspondence to: Dr. Marie Pier Desjardins, Division of Emergency Medicine, Department of Pediatrics, CHU Sainte-Justine, 3175 Chemin Côte Sainte-Catherine, Montreal, OC H3T 1C5; Email: mpdesj@gmail.com. 
possibilité d'élaborer un programme uniforme de formation partout au pays.
Keywords: point-of-care ultrasound, POCUS, pediatric emergency medicine fellowship, training, survey

\section{INTRODUCTION}

Emergency physicians have used point-of-care ultrasound (POCUS) for many years and for a variety of indications. ${ }^{1-5}$ More recently, POCUS has been integrated into pediatric emergency medicine (PEM) practice. ${ }^{6-10}$ The technology is appealing for PEM because it is usually painless, radiation free, potentially accessible, and valuable for augmenting both clinical decision-making and therapeutic intervention.

In 2012, the Canadian Association of Emergency Physicians (CAEP) published a renewed position statement, supporting emergency physicians in their use of POCUS. ${ }^{11}$ In the same year, Kim et al. published a survey of POCUS training in Canadian emergency medicine residency programs. ${ }^{12}$ Ninety-three percent of programs included formal POCUS training at the time of the study. All of these programs were offering training in focused assessment with sonography for trauma (FAST), cardiac assessment, and abdominal aortic aneurysm evaluation. The majority of centres included intrauterine pregnancy and procedural guidance training.

A POCUS Policy Statement and Technical Report by the American Academy of Pediatrics was recently published ${ }^{13}$ supporting POCUS use and quality control in pediatric emergency departments (EDs). These documents emphasize the need for formal POCUS education during PEM fellowship training. This sentiment is shared by the Royal College of Physicians and Surgeons of Canada, which is expected to add POCUS to the next iteration of its PEM curriculum. ${ }^{6}$

Surveys of American PEM training have demonstrated increasing inclusion of POCUS training in the last 5 years with $88 \%$ of programs reporting inclusion in 2012. ${ }^{14}$ The current state of adoption in Canadian PEM training programs remains unknown. To our knowledge, no study has described the current state of POCUS training in Canadian PEM programs or the perspectives of both program directors (PDs) and PEM fellows regarding POCUS technology and its clinical importance.

\section{METHODS}

This study consisted of two self-administered electronic mail surveys specifically designed for two subgroups of participants: Canadian PEM fellowship PDs and Canadian PEM fellows actively training. Surveys were disseminated electronically to PEM fellows of all 10 Canadian PEM fellowship programs through their PDs in 2014.

Three study investigators developed the surveys, one designed for PDs and the other for fellows. The surveys contained 20 (fellowship directors) and 21 (fellows) questions, respectively. The questions were formulated as multiple-choice response, with some exceptions (Likert scale and open questions).

To improve reliability and enhance clarity of the items, the survey was pilot tested - the PD survey by two former PDs at a single site, and the fellow survey by a group of recent PEM fellowship graduates. After refinement, the surveys were posted on a Web-based survey site (www.surveymonkey.com). The PDs of all 10 Canadian PEM fellowship programs were approached in person in January 2014 by one of the co-authors (BC). From April 2014 until October 2014, a modified Dillman's Tailored Design Method was used to enhance response. We sent repeated emails to the PDs, explaining the study, asking them to respond to the director's survey and to pass the link of the fellow's survey to their fellows. All participants were ensured of confidentiality and were provided instructions for survey completion. Data were collected electronically and analysed in an Excel spreadsheet (Microsoft, Richmond, WA). For analysis, we calculated proportions for each outcome.

The primary outcomes were the proportion of PEM programs that offers a formal curriculum in ultrasound (PD survey) and the training received by fellows during their fellowship (fellow survey). Secondary outcomes were 1) to describe current training in POCUS in PEM fellowship programs, 2) to compare what kind of training that PEM programs offer with what fellows actually receive, and 3) a needs assessment by fellows and PDs for the content of future training programs in POCUS.

The protocol was accepted by the Institutional Review Board of the CHU Sainte-Justine Research Institute. 
Informed consent was implied for participants who agreed to respond to the survey.

\section{RESULTS}

A total of $9 / 10(90 \%)$ of PDs and $42 / 60(70 \%)$ of PEM fellows responded to the survey. The responses represented all Canadian PEM training centres, except one.

Twenty-nine out of 41 (70\%) of the PEM fellows reported having no POCUS training prior to their fellowship and 28/38 (74\%) had POCUS training during fellowship. Among them, 13/38 (33\%) reported pediatric-specific POCUS training. A formal curriculum in POCUS was established in 5/9 (56\%) PEM programs as per the PDs, but only three programs offered PEM-specific training. All five programs established their training programs in 2011 or 2012. The applications included in the training programs varied between centres. The FAST and focused cardiac assessment were the most frequently taught (Table 1).

Twenty out of $40(50 \%)$ fellows reported that their peer fellows use POCUS at least once a week, and 4/9 (44\%) PDs reported the same; 7/9 (78\%) of PDs and $20 / 40(50 \%)$ of fellows stated that the majority of faculty rarely or never uses POCUS in clinical practice. But, only 11/39 (28\%) of fellows and 4/9 (44\%) of PDs stated that fellows rarely or never use it.

The FAST examination was the most commonly used clinical application (44\% of the PDs and $64 \%$ of the fellows responded that it is used most of the time or always). Other indications were rarely applied for POCUS use: only three fellows responded that it is used most of the time for cardiac, pulmonary, and musculoskeletal assessment, and one PD reported

\begin{tabular}{|c|c|c|c|}
\hline & \multicolumn{2}{|c|}{ Fellows $(n=38$ ) } & \multirow[b]{2}{*}{ No training } \\
\hline & Formal & Informal & \\
\hline FAST & 26 & 6 & 6 \\
\hline Cardiac assessment & 16 & 14 & 8 \\
\hline Pulmonary assessment & 9 & 19 & 10 \\
\hline Abdominal assessment & 8 & 17 & 13 \\
\hline Identify intrauterine pregnancy & 11 & 11 & 16 \\
\hline Guide procedures & 4 & 25 & 9 \\
\hline Identify musculoskeletal problems & 3 & 25 & 10 \\
\hline
\end{tabular}

that POCUS was mostly used for pulmonary and abdominal assessment.

Eighty-six percent of fellows and $80 \%$ of PDs stated that training in POCUS was very important or essential for PEM training. Both fellows and PDs responded in favor of incorporating formal POCUS training into their PEM program (Figure 1). However, fellows ranked POCUS to be comparatively more important than other core curriculum elements when contrasted with PD responses. We asked them to compare the relative importance of different procedural interventions during their fellowship: POCUS, resuscitation, trauma, orthopedic techniques (e.g., fracture reduction), and sedation/nerve blocks, using a Likert scale of 1 ("not important") to 5 ("very important"). No PD reported the actual importance of POCUS as 5, and one stated that POCUS should have an importance of 5. When asking fellows the same question, results were different: $19 \%$ reported the actual importance as 5 , and $46 \%$ stated that POCUS should have that maximal state of importance. These results were comparable to the importance given to procedural sedation and nerve blocks as far as fellows were concerned ( $49 \%$ gave it a 5 on the Likert scale for the importance it should have), whereas PDs rated sedation higher than POCUS (44\%). Fellows as well as PDs rated resuscitation, trauma, and techniques more important than POCUS during fellowship training: fellows gave it a 5 on the Likert scale in $89 \%, 86 \%$, and $62 \%$, respectively, and for PDs the numbers were $89 \%, 89 \%$, and $67 \%$.

Fellows and PDs stated that the major barriers to training were lack of trained faculty $(95 \%$ and $90 \%$, respectively), followed by insufficient faculty time $(62 \%$ and $67 \%$ ), fellows' time (49\% and $44 \%$ ), and faculty interest in the technology ( $41 \%$ and $44 \%$ ) (Figure 2). PDs of all nine programs reported access to an ultrasound machine in their ED. Seven ED settings owned their own ultrasound system, and one was planning to buy a system imminently.

\section{DISCUSSION}

POCUS has become an important adjunct in the pediatric ED and is gaining more and more importance in PEM practice and fellowship training. ${ }^{8,11,14}$ However, the integration of POCUS in Canada still lags behind the U.S. PEM fellowship programs. Ramirez et al. (2006) and Marin et al. (2011) queried all U.S. PEM fellowship directors with surveys concerning 
(a)

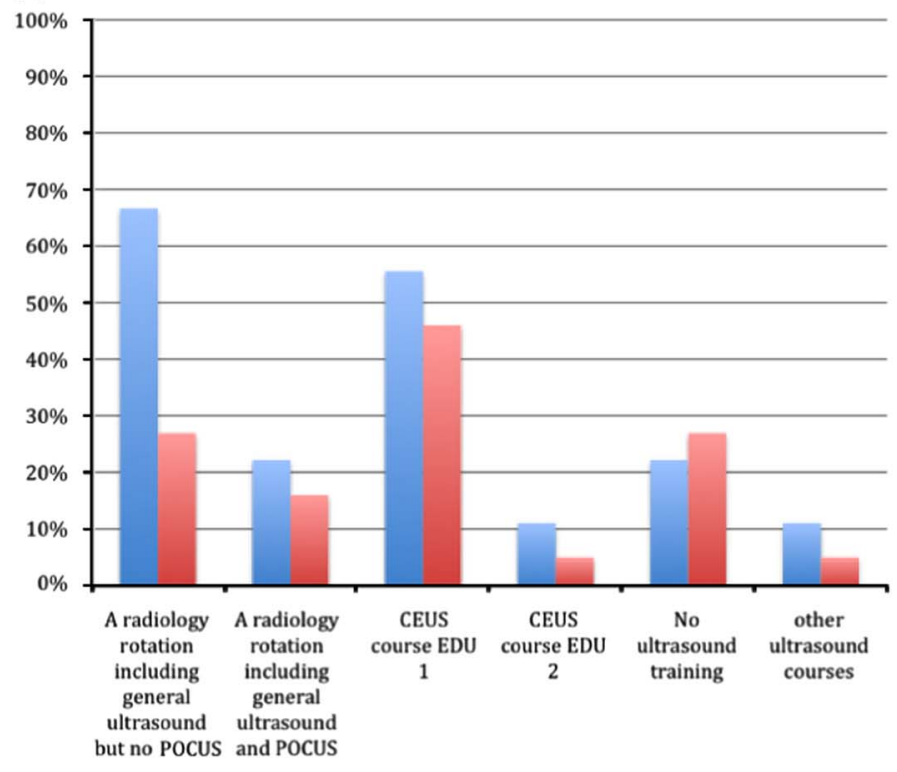

(b)

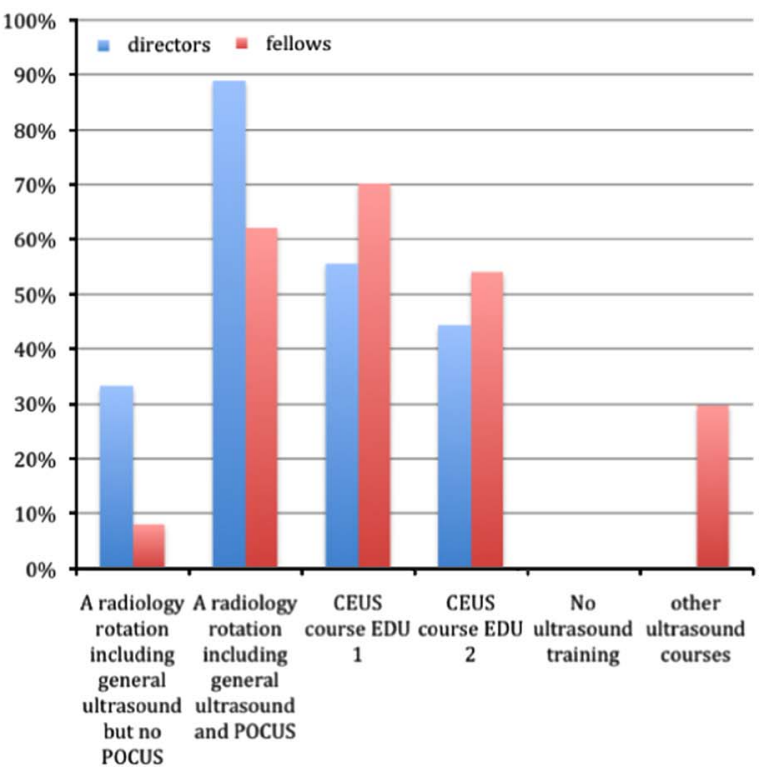

Figure 1. The following training in Medical Imaging a) is part of our program and b) should be part of the program in the future (other: ECCU or equivalent, pediatric specific Canadian Society of Emergency Ultrasound (CEUS) course, specific pediatric POCUS course, video quiz, module-based programming).

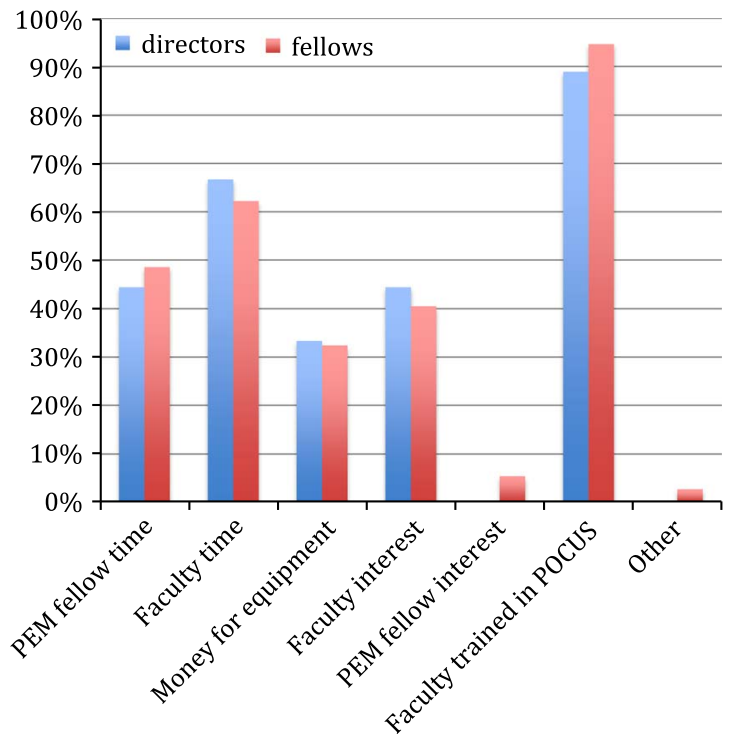

Figure 2. "Important barriers in learning POCUS during my PEM fellowship is that there is not enough. .."

this subject, and, in 2008, Chamberlain et al. sent a survey to all U.S. pediatric emergency medical directors, fellowship directors, and graduating fellows. ${ }^{14-16}$ In 2011, 53/60 (88\%) of U.S. PEM programs responding to their survey offer some POCUS training to their fellows, ${ }^{14}$ compared to $65 \%$ in $2006^{15}$ and $56 \%$ of the Canadian programs in our study. A pediatric-specific
POCUS curriculum is currently offered in only three (33\%) of the Canadian PEM programs, which is similar to U.S. reporting 5 years ago. ${ }^{15}$

Marin et al. ${ }^{14}$ reported $63 \%$ use of FAST ("most of the time" or "always") in U.S. hospitals with a pediatric EM training program, compared to $50 \%$ in our study. In the study by Chamberlain et al., FAST use was reported by fellows to be $93 \% .{ }^{16}$ In that same survey, $53 \%$ of responders (fellows, PDs, and medical directors) stated that POCUS was used for soft tissue evaluation in their ED, 37\% stated its use for cardiac assessment, 9\% for abdominal assessment, and $40 \%$ for procedural guidance. In our study, the vast majority of PEM fellows stated that POCUS was used for these indications "never," "rarely", or "sometimes" (36 fellows for musculoskeletal problems, 35 for cardiac, 39 for abdominal assessment, and 36 for procedural guidance).

Fellows in our study were more enthusiastic about use of POCUS in their EDs than their PDs. A potential explanation for this difference might be that PDs underestimate the use of POCUS, whereas fellows have broader insight to daily practice because they work directly with a great number of staff. The fellows' presence might also stimulate staff to use POCUS more often.

Fellows in general believed POCUS to be more important than their PDs. This difference could be 
explained by a number of factors, including the fellows' clinical experience with the technology, their understanding of the literature, their comfort with technology, or the sophistication and growth of their clinical practice. One of the PDs commented: "I am not convinced that POCUS, despite it being sexy and fun, has really been shown to improve patient care in a pediatric setting." Although this might represent a familiar sentiment, the body of literature to support the use of POCUS in PEM is becoming more and more robust and includes literature that supports the use of POCUS for assessment of heart and lung pathologies, intussusception, hypertrophic pyloric stenosis, appendicitis, long bone fractures, skull fractures, skin and soft tissue infection, and procedural guidance. ${ }^{7-9,17}$

The majority of fellows stated that they would like to incorporate a CEUS course $(70 \%$ for emergency department echography [EDE] 1, 54\% for EDE 2) as part of their POCUS curriculum, whereas 30\% asked for "other" courses (the latter included pediatric POCUS courses). This response suggests that the majority of sites still lack the capacity to incorporate POCUS into their curriculum due to a lack of local expertise or instructional infrastructure and are dependent on third-party training. However, PEM POCUS fellowship programs that are developing leaders in PEM POCUS may soon remedy this issue. ${ }^{6}$

The fact that FAST examination is the indication most commonly used and taught is not surprising. The FAST examination is part of the Advanced Trauma Life Support (ATLS) program and one of the original POCUS indications. However, its use and impact in the pediatric setting is still unclear and has been shown to be less helpful than in adult counterparts. ${ }^{10}$ Curricular development should include specific pediatric considerations rather than "adopting" adult training.

Some of the barriers identified in our study are of temporary nature, and integration of POCUS training in Canada PEM fellowships should mirror the impressive adoption seen in U.S. programs in the near term. ${ }^{14}$ For example, the use of external resources can negate the current lack of trained faculty and bridge programs until local faculty is adequately trained to supervise fellows. Both CCFP-EM and FRCPC have traditionally relied on EDE programs for all POCUS training, but they slowly are transitioning to more site-specific content as a result of more fellowship-trained POCUS faculty (e.g., University of Toronto). Similarly, the perception that POCUS impedes workflow may reflect a lack of faculty experience and the lack of infrastructure that promotes timely and accessible use of POCUS. More challenging is the lack of time available in many curriculums for POCUS training. Only with greater recognition and supporting evidence can POCUS be expected to replace other elements of training.

Both PEM fellows and PDs agree that POCUS is very important or essential for PEM training. This suggests that the majority of fellowship programs would welcome a PEM-specific curriculum, as well as materials for training and tools for assessing competency.

\section{LIMITATIONS}

The major limitation of this study is the small sample size. However, we did receive responses from a high proportion of possible respondents. Another limitation was that PDs had to pass the survey to their fellows. We chose this way of communication due to that there is no database to allow us to access fellows directly recognizing that it could lead to bias. Also, responders were likely more interested in the subject than those who did not answer. Similarly, some sites had several fellows responding, whereas others had very few, making it difficult to interpret the result as being representative of all Canadian PEM fellows. Still, the response rate of 9 of 10 PDs and $70 \%$ of actual training fellows suggests a reasonable description. Finally, we wanted to keep the results anonymous, and, because some programs are very small, we were not able to differentiate fellows per site and remove regional bias.

\section{CONCLUSION}

PEM fellows and PDs agree on the importance of POCUS in PEM and the need to reprioritize this training. There is a wide variation in the execution and content of the training that does exist, with only $56 \%$ of Canadian PEM fellowship programs offering integrated POCUS training as part of their curriculum. The greatest barrier appears to be a lack of trained faculty to teach. The opportunity exists to develop a standardized curriculum as well as to augment faculty-training capacity across Canada.

Acknowledgements: The authors would like to thank all participants of this survey. 
Competing interests: None declared.

\section{REFERENCES}

1. Jehle D, Davis E, Evans T, et al. Emergency department sonography by emergency physicians. Am $\mathcal{f}$ Emerg Med 1989;7(6):605-11.

2. American College of Emergency Physicians (ACEP). American College of Emergency Physicians Policy Statement: Emergency Ultrasound Guidelines; October 2008. Available at: http://www. acep.org/WorkArea/DownloadAsset.aspx?id=32878.

3. Labovitz AJ, Noble VE, Bierig M, et al. Focused cardiac ultrasound in the emergent setting: a consensus statement of the American Society of Echocardiography and American College of Emergency Physicians. $\mathcal{F}$ Am Soc Echocardiogr 2010;23(12):1225-30.

4. Moore CL, Gregg S, Lambert M. Performance, training, quality assurance, and reimbursement of emergency physician-performed ultrasonography at academic medical centers. 7 Ultrasound Med 2004;23(4):459-66.

5. Schlager D, Lazzareschi G, Whitten D, et al. A prospective study of ultrasonography in the ED by emergency physicians. Am 7 Emerg Med 1994;12(2):185-9.

6. Rosenfield D, Kwan C, Fischer J. Point-of-care ultrasound: an emerging technology in Canadian paediatrics. Paediatr Child Health 2015;20(2):67-8.

7. Chen L, Baker MD. Novel applications of ultrasound in pediatric emergency medicine. Pediatr Emerg Care 2007; 23(2):115-26.

8. Gallagher RA, Levy JA. Advances in point-of-care ultrasound in pediatric emergency medicine. Curr Opin Pediatr 2014;26(3):265-71.
9. Levy JA, Bachur RG. Bedside ultrasound in the pediatric emergency department. Curr Opin Pediatr 2008;20(3): 235-42.

10. Scaife ER, Rollins MD, Barnhart DC, et al. The role of focused abdominal sonography for trauma (FAST) in pediatric trauma evaluation. $\mathcal{F}$ Pediatr Surg 2013; 48(6):1377-83.

11. Henneberry RJ, Hanson A, Healey A, et al. Use of point of care sonography by emergency physicians. CFEM 2012; 14(2):106-12.

12. Kim DJ, Theoret J, Liao MM, et al. The current state of ultrasound training in Canadian emergency medicine programs: perspectives from program directors. Acad Emerg Med 2012;19(9):e1073-8.

13. Marin JR, Lewiss RE, American Academy of Pediatrics, Committee on Pediatric Emergency Medicine, et al. Pointof-care ultrasonography by pediatric emergency medicine physicians. Pediatrics 2015;135(4):e1097-104.

14. Marin JR, Zuckerbraun NS, Kahn JM. Use of emergency ultrasound in United States pediatric emergency medicine fellowship programs in 2011. F Ultrasound Med 2012; 31:1357-63.

15. Ramirez-Schrempp D, Dorfman DH, Tien I, Liteplo AS. Bedside ultrasound in pediatric emergency medicine fellowship programs in the United States: little formal training. Pediatr Emerg Care 2008;24(10):664-7.

16. Chamberlain MC, Reid SR, Madhok M. Utilization of emergency ultrasound in pediatric emergency departments. Pediatr Emerg Care 2011;27(7):628-32.

17. Leeson K, Leeson B. Pediatric ultrasound: applications in the emergency department. Emerg Med Clin North Am 2013;31(3):809-29. 